\title{
Rezensionen online
}

\section{... von der Redaktion betreut (September-Oktober 2020)}

Die Redaktion der Vierteljahrshefte für Zeitgeschichte arbeitet seit 2003 im Auftrag des Instituts für Zeitgeschichte München-Berlin mit dem Rezensionsjournal sehepunkte zusammen. Diese Kooperation findet nicht nur in den sehepunkten ihren Niederschlag, sondern auch in den Vierteljahrsheften selbst: In jedem Heft werden die von der Redaktion angeregten und betreuten Rezensionen angezeigt, die in den Monaten zuvor in den sehepunkten erschienen sind.

Omri Boehm, Israel - eine Utopie, Berlin 2020.

Rezensiert von: Tamar Amar-Dahl (Berlin) in sehepunkte 20 (2020), Nr. 9

www.sehepunkte.de/2020/09/34687.html

Michael Borchard u. a. (Hgg.), Entspannung im Kalten Krieg. Der Weg zum Moskauer Vertrag und zur KSZE, Graz 2020.

Rezensiert von: Bernd Rother (Bundeskanzler-Willy-Brandt-Stiftung, Berlin) in sehepunkte 20 (2020), Nr. 9

www.sehepunkte.de/2020/09/34688.html

Eileen Boris, Making the Woman Worker. Precarious Labor and the Fight for Global Standards, 1919-2019, New York 2019.

Rezensiert von: Manuela Rienks (Institut für Zeitgeschichte München-Berlin) in sehepunkte 20 (2020), Nr. 9

www.sehepunkte.de/2020/09/33949.html

Günter Buchstab (Bearb.), Kohl: „Gelassenheit und Zuversicht“. Die Protokolle des CDU-Bundesvorstands 1980-1983, Düsseldorf 2018.

Rezensiert von: Thomas Schlemmer (Institut für Zeitgeschichte München-Berlin) in sehepunkte 20 (2020), Nr. 9

www.sehepunkte.de/2020/09/33177.html

Jeffrey James Byrne, Mecca of Revolution. Algeria, Decolonization, and the Third World Order, New York 2016.

Rezensiert von: Jéronimo L. S. Barbin (Zentrum für Militärgeschichte und Sozialwissenschaften der Bundeswehr, Potsdam) in sehepunkte 20 (2020), Nr. 9

www.sehepunkte.de/2020/09/30256.html

Paul Collins / Charles Tripp (eds.), Gertrude Bell and Iraq. A Life and Legacy, Oxford 2017.

Rezensiert von: Bernd Lemke (Zentrum für Militärgeschichte und Sozialwissenschaften der Bundeswehr, Potsdam) in sehepunkte 20 (2020), Nr. 9

www.sehepunkte.de/2020/09/34132.html 
Huw Dylan / David Gioe / Michael S. Goodman, The CIA and the Pursuit of Security. History, Documents and Contexts, Edinburgh 2020.

Rezensiert von: Thomas Riegler (Wien) in sehepunkte 20 (2020), Nr. 10

www.sehepunkte.de/2020/10/34603.html

Astrid M. Eckert, West Germany and the Iron Curtain. Environment, Economy, and Culture in the Borderlands, New York 2019.

Rezensiert von: Pascal Pawlitta (Institut für Zeitgeschichte München-Berlin) in sehepunkte 20 (2020), Nr. 10

www.sehepunkte.de/2020/10/33950.html

Hagen Fleischer, Krieg und Nachkrieg. Das schwierige deutsch-griechische Jahrhundert, Wien / Köln / Weimar 2020.

Rezensiert von: Loukas Lymperopoulos (Hamburg) in sehepunkte 20 (2020), Nr. 10

www.sehepunkte.de/2020/10/34550.html

Swantje Greve, Das „System Sauckel“. Der Generalbevollmächtigte für den Arbeitseinsatz und die Arbeitskräftepolitik in der besetzten Ukraine 1942-1945, Göttingen 2019.

Rezensiert von: Daniel Uziel (Yad Vashem / Ben Gurion University) in sehepunkte 20 (2020), Nr. 9

www.sehepunkte.de/2020/09/33327.html

Maddalena Guiotto / Helmut Wohnout (Hgg.), Italien und Österreich im Mitteleuropa der Zwischenkriegszeit / Italia e Austria nella Mitteleuropa tra le due guerre mondiali, Wien / Köln / Weimar 2018.

Rezensiert von: Michael Gehler (Hildesheim) in sehepunkte 20 (2020), Nr. 9

www.sehepunkte.de/2020/09/33871.html

Wolfgang Hien, Die Arbeit des Körpers. Eine kritische Arbeitsgeschichte von der Hochindustrialisierung in Deutschland und Österreich bis zur neoliberalen Gegenwart, Wien 2018.

Rezensiert von: Sebastian Voigt (Institut für Zeitgeschichte München-Berlin) in sehepunkte 20 (2020), Nr. 9

www.sehepunkte.de/2020/09/34334.html

Jonathan Hogg, British Nuclear Culture. Official and Unofficial Narratives in the Long 20th Century, London u. a. 2016.

Rezensiert von: Mathias Häußler (Universität Regensburg) in sehepunkte 20 (2020), Nr. 9 www.sehepunkte.de/2020/09/30550.html 
Christian Ingrao, The Promise of the East. Nazi Hopes and Genocide, 1939-43, Cambridge / Medford, MA 2019.

Rezensiert von: Alexa Stiller (Universität Bern / University of Oxford) in sehepunkte 20 (2020), Nr. 10

www.sehepunkte.de/2020/10/34371.html

Wolfgang Jäger, Soziale Bürgerrechte im Museum. Die Repräsentation sozialer Demokratie in neun kulturhistorischen Museen, Bielefeld 2020.

Rezensiert von: Julian Genten (Friedrich-Meinecke-Institut, Freie Universität Berlin) in sehepunkte 20 (2020), Nr. 10

www.sehepunkte.de/2020/10/34435.html

Wolfgang Jäger, Soziale Sicherheit statt Chaos. Beiträge zur Geschichte der Bergarbeiterbewegung an der Ruhr, Essen 2018.

Rezensiert von: Jan De Graaf (Leuven / Bochum) in sehepunkte 20 (2020), Nr. 9

www.sehepunkte.de/2020/09/32860.html

Chen Jian u. a. (eds.), The Routledge Handbook of the Global Sixties. Between Protest and Nation-Building, London / New York 2018.

Rezensiert von: Philipp Gassert (Universität Mannheim) in sehepunkte 20 (2020), Nr. 10

www.sehepunkte.de/2020/10/32021.html

Hannah Jonas, Fußball in England und Deutschland von 1961 bis 2000. Vom Verlierer der Wohlstandsgesellschaft zum Vorreiter der Globalisierung, Göttingen 2019.

Rezensiert von: Nils Havemann (Historisches Institut, Universität Stuttgart) in sehepunkte 20 (2020), Nr. 10

www.sehepunkte.de/2020/10/34151.html

Nina Kleinöder / Stefan Müller / Karsten Uhl (Hgg.), „Humanisierung der Arbeit“. Aufbrüche und Konflikte in der rationalisierten Arbeitswelt des 20. Jahrhunderts, Bielefeld 2019.

Rezensiert von: Jacopo Ciammariconi (Universität Trier) in sehepunkte 20 (2020), Nr. 10

www.sehepunkte.de/2020/10/33909.html

Inga Markovits, Diener zweier Herren. DDR-Juristen zwischen Recht und Macht, Berlin 2020.

Rezensiert von: Hermann Wentker (Institut für Zeitgeschichte München-Berlin) in sehepunkte 20 (2020), Nr. 9

www.sehepunkte.de/2020/09/34604.html

Siegfried Mielke / Stefan Heinz, Alwin Brandes (1866-1949). Oppositioneller Reformer - Widerstandskämpfer, Berlin 2019.

Rezensiert von:Jens Becker (Hans-Böckler-Stiftung, Düsseldorf) in sehepunkte 20 (2020), Nr. 10 www.sehepunkte.de/2020/10/33579.html 
Christian Möller, Umwelt und Herrschaft in der DDR. Politik, Protest und die Grenzen der Partizipation in der Diktatur, Göttingen 2020.

Rezensiert von: Tobias Huff (Johannes Gutenberg-Universität, Mainz) in sehepunkte 20 (2020), Nr. 9

www.sehepunkte.de/2020/09/34100.html

Rezensiert von: Sophie Lange (Berliner Kolleg Kalter Krieg am Institut für Zeitgeschichte München-Berlin) in sehepunkte 20 (2020), Nr. 9

www.sehepunkte.de/2020/09/33628.html

Michael Cotey Morgan, The Final Act. The Helsinki Accords and the Transformation of the Cold War, Princeton / Oxford 2018.

Rezensiert von: Wilfried von Bredow (Philipps-Universität, Marburg) in sehepunkte 20 (2020), Nr. 10

www.sehepunkte.de/2020/10/32983.html

Markus Nesselrodt, Dem Holocaust entkommen. Polnische Juden in der Sowjetunion, 1939-1946, Berlin / Boston 2019.

Rezensiert von: Andrea Löw (Zentrum für Holocaust-Studien am Institut für Zeitgeschichte, München) in sehepunkte 20 (2020), Nr. 10

www.sehepunkte.de/2020/10/33874.html

Xosé M. Núñez Seixas, Die bewegte Nation. Der spanische Nationalgedanke 18082019, Hamburg 2019.

Rezensiert von: Reiner Tosstorff (Johannes Gutenberg-Universität, Mainz) in sehepunkte 20 (2020), Nr. 10

www.sehepunkte.de/2020/10/33557.html

Martin Rempe, Kunst, Spiel, Arbeit. Musikerleben in Deutschland, 1850 bis 1960, Göttingen 2020.

Rezensiert von: Friedemann Pestel (Historisches Seminar, Albert-Ludwigs-Universität, Freiburg/Brsg.) in sehepunkte 20 (2020), Nr. 10

www.sehepunkte.de/2020/10/33845.html

David Rousset, Das KZ-Universum, Berlin 2020.

Rezensiert von: Nina Rabuza (München / Berlin) in sehepunkte 20 (2020), Nr. 9

www.sehepunkte.de/2020/09/34472.html

Edith Schriefl, Versammlung zum Konsens. Der sächsische Landtag 1946-1952, Ostfildern 2020.

Rezensiert von: Jürgen John (Jena) in sehepunkte 20 (2020), Nr. 10

www.sehepunkte.de/2020/10/34599.html 
Richard Smith (ed.), Britain and the Revolutions in Eastern Europe, 1989, London / New York 2020.

Rezensiert von: Jost Dülffer (Köln) in sehepunkte 20 (2020), Nr. 9

www.sehepunkte.de/2020/09/33624.html

Katharina Steidl, Am Rande der Fotografie. Eine Medialitätsgeschichte des Fotogramms im 19. Jahrhundert, Berlin / Boston 2019.

Rezensiert von: Olaf Kistenmacher (Hamburg) in sehepunkte 20 (2020), Nr. 10

www.sehepunkte.de/2020/10/34698.html

Enric Ucelay-Da Cal / Arnau Gonzàlez i Vilalta / Xosé M. Núñez Seixas (eds.), El catalanisme davant del feixisme (1919-2018), Maçanet de la Selva 2018.

Rezensiert von: Reiner Tosstorff (Johannes Gutenberg-Universität, Mainz) in sehepunkte 20 (2020), Nr. 10

www.sehepunkte.de/2020/10/33557.html

Alexander Vazansky, An Army in Crisis. Social Conflict and the U.S. Army in Germany, 1968-1975, Lincoln 2019.

Rezensiert von: Christian Th. Müller (Potsdam) in sehepunkte 20 (2020), Nr. 9

www.sehepunkte.de/2020/09/33754.html

Elizabeth White, A Modern History of Russian Childhood. From the Late Imperial Period to the Collapse of the Soviet Union, London u. a. 2020.

Rezensiert von: Yuliya von Saal (Institut für Zeitgeschichte München-Berlin) in sehepunkte 20 (2020), Nr. 9

www.sehepunkte.de/2020/09/34493.html

\section{Rezensionen zu Publikationen des IfZ (September-Oktober 2020):}

Thorsten Holzhauser, Die „Nachfolgepartei“. Die Integration der PDS in das politische System der Bundesrepublik Deutschland 1990-2005, Berlin / Boston 2019. Rezensiert von: Till Kössler (Martin-Luther-Universität, Halle-Wittenberg) in sehepunkte 20 (2020), Nr. 9

www.sehepunkte.de/2020/09/33837.html

Petra Weber, Getrennt und doch vereint. Deutsch-deutsche Geschichte 19451989/90, Berlin 2020.

Rezensiert von: Arnd Bauerkämper (Friedrich-Meinecke-Institut, Freie Universität Berlin) in sehepunkte 20 (2020), Nr. 10

www.sehepunkte.de/2020/10/34332.html 